\title{
Assessment of Videoendoscopy-assisted Abdominoplasty for Diastasis Recti Patients
}

\author{
Cheng-Jen Chang
}

Background: The objective of this retrospective analysis was to assess the treatment of endoscope abdominoplasty for diastasis recti deformity patients.

Methods: $\quad$ From January 1999 to January 2011, 88 patients ranging from 35 to 46 years in age were treated with videoendoscopy-assisted minimally invasive surgery. All patients were Asian. Early ( $<3$ months) and late ( $>6$ months) complications were assessed throughout a follow-up period of up to 66 months.

Results: Observations were conducted at the end of three weeks, six months, and 66 months. Early on, all patients experienced numbness with local paresthesia (100\%) closely after treatment, and reported the feelings to subside by six months post-treatment. Four patients $(4.5 \%)$ experienced ecchymosis, and three patients $(3.4 \%)$ were affected by seroma. One patient $(1.1 \%)$ had dyspnea immediately after surgery, which recovered after oxygen $\left(\mathrm{O}_{2}\right)$ administration. Only one patient $(1.1 \%)$ experienced minimal skin loss, which recovered after 3 months of surgery, and there were no further complications. Hypertrophic scars were apparent in three patients $(3.4 \%)$ who showed no unwanted signs or further complications after post-operative scar care. No hematoma had been reported.

\section{At a Glance Commentary \\ Scientific background of the subject}

The goals of standard abdominoplasty are to remove excess skin and fat, contour the abdomen and adjunct hip regions, and strengthen any significant weakness of the abdominal wall. However, the postoperative scar sometime is unacceptaly. The use of the endoscopic techniques has been a significant turnaround from the traditional techniques.

\section{What this study adds to the field}

Endoscopy abdominoplasty may be indicated for diastasis recti deformity with minimal excess skin and fat. Meanwhile, controlled dermal injury may contract enough skin to allow the use of this technique in patients with a moderate excess of skin. Advantages include: Minimal scarring, less numbness, acceptability, less bleeding, and less edema.

Conclusions: Videoendoscopy-assisted abdominoplasty can be used for diastasis recti deformity with minimal excess skin. Our study demonstrated effects against the formation of seroma and other complications. (Biomed J 2013;36:252-256)

Key words: abdominoplasty, diastasis recti, videoendoscopy

$\mathrm{T}_{\mathrm{i}}^{\mathrm{s}}$ The most significant characteristic of using an endoscope in surgery is that the treatment becomes minimally invasive, resulting in the reduction of bleeding. ${ }^{[1-10]}$ Endoscopes can form curves successfully only if patients fall under certain indications. Excess skin is only one of the indications in which quantity can play a significant role in determining the optimal results of endoscopic procedures. Most Asian women have post-pregnancy stretched muscle/skin tissue with less excessive skin or subcutaneous fatty tissue. Previous studies show the endoscope to provide overwhelming results in various abdominoplasty research studies and applications. ${ }^{[11-14]}$ The objective of this retrospective study is to evaluate the videoendoscopy-assisted abdominoplasty for diastasis recti deformity with minimal invasive surgery.

From the Aesthetic Medical Center, Department of Plastic Surgery, Chang Gung Memorial Hospital at Taipei, Chang Gung University College of Medicine, Taoyuan, Taiwan

Received: May 8, 2012; Accepted: Sep. 24, 2012

Correspondence to: Dr. Cheng-Jen Chang, Department of Plastic Surgery, Chang Gung Memorial Hospital at Taipei. 199, Dunhua N. Rd., Songshan Dist., Taipei 105, Taiwan (R.O.C.). Tel: 886-2-27135211 ext. 3502; Fax: 886-2-25140600; E-mail: cheng.jen@adm.cgmh.org.tw

DOI: $10.4103 / 2319-4170.113374$ 


\section{METHODS}

Between January 1999 and January 2011, 88 female patients were evaluated and chosen to take part in our study based on the classified appearance and type of their post-pregnancy stretched muscle/skin tissue. Our patients were selected according to the following criteria: (1) patient was not accepting of the scar from an open abdominoplasty; (2) patient needed a muscle-fascia repair without panniculectomy; (3) patient could benefit from the repair having enough diastasis recti and fascial laxity; (4) patient's abdominal skin would shrink without associated excision of extra skin and/or with liposuction in a preoperative evaluation, (5) patients' body mass index (BMI) ranged between 24 and 35. Patients with stretched muscle/skin tissue can be categorized into four classes: Type A, where stretching occurs at the junction of the rectus sheath at the center of the abdominal area; Type B, having laxity of the central and lateral abdominal area, along with the infraumbilical aponeurotic layer at either lateral side; Type $\mathrm{C}$ involving the congenital lateral insertion of the recti muscles due to the outward spreading of muscle tissue; and Type D where there is an advanced spreading of muscle tissue, along with minimal support of muscle tissue along the patient's waistline. This review will refer to the patients according to their classification group, which helped determine the endoscopic abdominoplasty procedure they underwent.

All patients were evaluated and treated by a single surgeon (C-J, C). Preoperative work included routine lab work, electrocardiogram, and a chest radiograph on every patient. Human immunodeficiency virus test was also administered to each patient. One day before the surgery, the patients had a consultation and discussion with the anesthesiologist about the details of general anesthesia. While photos and video were taken and administered preoperatively, photos were also taken of the patients during the follow-up periods. A videoendoscope with connected monitor was used to display each step of procedure. The endoscope was $10 \mathrm{~mm}$ in diameter, with an angle of 0 (Snoden- Pancer, U.S.A.). The basic medical instrumentation consisted of endoscopic elevators, nerve hooks, retractors, scissors, cautery, and needle holders. Suction of the smoke generated by cauterization was also necessary. Dissection was performed by relaying images from the endoscope to a monitor.

Technical treatment comprised of the beginning of patients' assessment, and preoperative markings of the treated area were made. Patients were asked to stand erect where the abdominal perimeter was marked from the costal margin (lower ribcage) of the right and left, to the top part of the xiphoid process of the sternum, then bilaterally along the anterior axillary lines, down to suprainguinal areas and bottom at suprapubic lines. Internal elliptical markings were also made on the protruded lines of the bilateral rectus abdominalis muscle. Thereafter, in order to lift pocket and apply plication (folding) of external layers of rectal sheaths of bilateral rectus abdominalis muscle, markings were also made on the mid-section [Figure 1].

Once our study's administrators were at their assigned stations according to the lithotomy/operative positions (starting at the head of the patient going clockwise: Anesthesiologist, nurse, surgeon, and assistant doctor), patients were given an endotracheal tube and general anesthesia. Hair over the abdomen and mons pubis was shaved and an indwelling Foley catheter was inserted. When the abdomen was prepped and draped for associate procedures of liposuction, a tumescent solution consisting of normal saline $300 \mathrm{ml}$, sodium bicarbonate $10 \mathrm{ml}, 1 \%$ xylocaine $(120 \mathrm{ml})$, and 1:100,000 epinephrine $(0.6 \mathrm{ml})$ was topically administered. After liposuction and body reshaping, a suprapubic incision was made below the hairline to omit visible scarring, with an incision length of no more than $5 \mathrm{~cm}$. The dissection plan was just above rectal sheath. Videoendoscopic visualization and insertion instruments for dissection were made. A periumbilical incision was made when dissection of upper abdominal area was indicated. During dissection at periumbilical area, finger insertion in umbilical fossa for prevention of umbilical injury was taken as well. Once external markings for dissection were complete, as discussed previously, internal stitching for plication of external rectal sheath was taken. A 2-0 Prolen was double enforced by first running sutures from the distal of suprapubic area to the proximal section of infraumbilical area, and then back down to distal for a closure tie to be made at the suprapubic area. For the same procedure, a 1-0 and/or 2-0 Prolen were used for double enforcement by

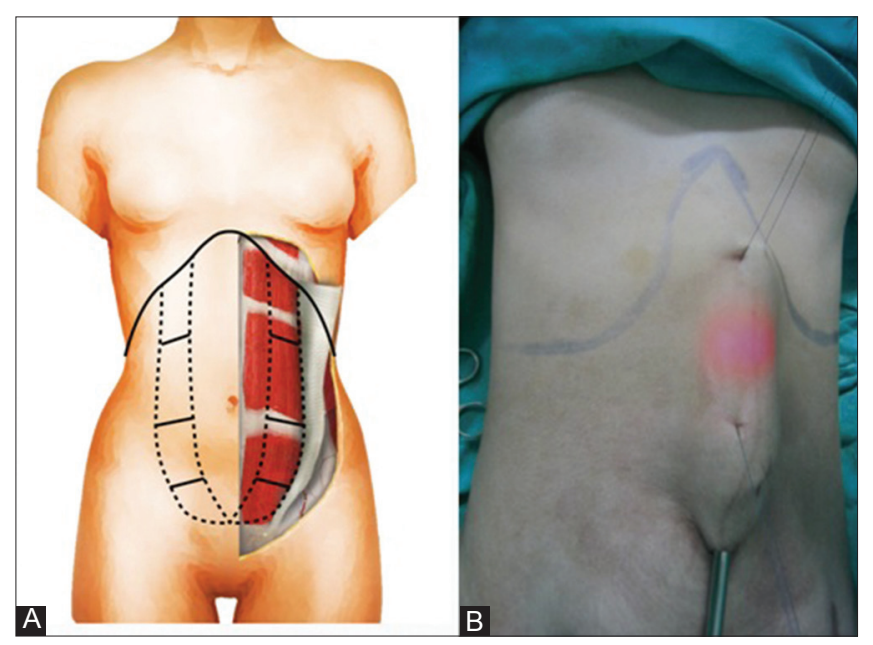

Figure 1: (A) Preoperative markings with abdominal perimeter marked, starting from costal margin to the right, then from top of xyphoid process to around left ribcage (dotted line). Marking for suprapubic incision was made at bottom for visualization and instruments. (B) Image of internal, mid-section lifted pocket. 
first running sutures from the distal of supraumbilical area to the proximal section of lower sternal area, and then back down to distal for a closure tie to be made at the supraumbilical area [Figure 2]. There was no need to drain any of the 88 patients. In our patient group, $16(18.1 \%)$ patients received extra procedures, all in the form of liposuction, which were administered for purposes of seeking complete body contouring and optimal reshaping. Extra procedures of augmentative mammoplasty for two (2.3\%) patients were also performed. Six patients $(6.8 \%)$ had $4 \mathrm{cc}$ of Fibrin sealant (Tissueco Duo, Immuno NV, 1060 Brussels) sprayed into the dissected pockets to seal the tissue. Postoperatively, a pressure abdominal garment was worn for about 3-6 weeks to provide external support.

Following appropriate abdominoplasty treatment, patients were admitted into the hospital for overnight care and observation. Where multiple procedures were scheduled, patients received extended care. All patients were closely observed and responses were recorded regularly for three weeks, three months, six months, and 66 months after treatment, at an average of 38 months.

\section{RESULTS}

Information regarding variables of age, severity of diastasis recti abdominis, results, and precluded side effects were recorded for analysis. All 88 patients fell under diastasis recti Type A, where stretching occurred in the junction of the rectus sheath at the center of the abdominal area. Their ages ranged from 35 to 46 years with an average age of 37 years. Patients' Body Mass Index (BMI) ranged between 24 and 35 with average of 32 years of age. The operative time ranged from one hour 50 minutes to three hours with the mean period of two hours 10 minutes. The admission period ranged from one to seven days, with an average of three days. Inpatient care was based on pres-

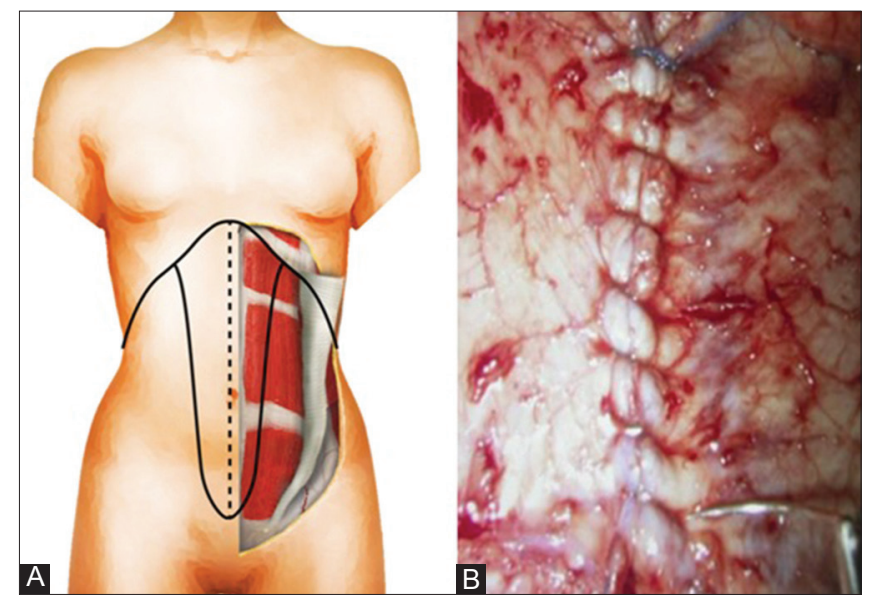

Figure 2: (A) Markings of applied plication of external rectal fascia and internal stitching marked by dotted line (B) Image of internal stitching. ence of early complications as well as wound care. Early follow-up was taken at three weeks, with further assessment at three months, six months, and the final results, observed and recorded at 66 months [Table 1].

In our early assessment at three months, all patients $(100 \%)$ collectively developed numbness together with local paresthesia. However such reactions subsided gradually amongst all patients at the six-month follow-up period. Nine patients $(10.2 \%)$ experienced ecchymosis and serums affected three patients $(3.4 \%)$. One patient $(1.1 \%)$ had dyspnea immediately after surgery, yet recovered after oxygen $\left(\mathrm{O}_{2}\right)$ administration. Only one patient $(1.1 \%)$ had minimal skin loss, but this patient recovered within three months following surgery and reported no further complications. Hematoma had been recorded as a presumable side effect, yet no patients produced signs of such reactions. Hypertrophic scars were apparent in three patients (3.4\%) yet showed no unwanted signs of treatment. All complications subsided ( $>6$ months) postoperatively until the 66-monthfinal follow-up period had arrived [Figure 3].

Table 1: Results and precluded side effects of 88 patients using videoendoscope-assisted abdominoplasty as in the study

\begin{tabular}{lccc}
\hline Side effects & $\begin{array}{c}\text { Early }(\%) \\
\text { [at 3 weeks] }\end{array}$ & $\begin{array}{c}\text { Late }(\%) \\
\text { [at 6 months] }\end{array}$ & $\begin{array}{c}\text { Final (\%) } \\
\text { [at 66 months] }\end{array}$ \\
\hline $\begin{array}{l}\text { Numbness and } \\
\text { Paraesthesia }\end{array}$ & $52(100 \%)$ & 0 & 0 \\
Ecchymosis & $9(10.2 \%)$ & 0 & 0 \\
Hypertrophic scar & $3(3.4 \%)$ & 0 & 0 \\
Seroma & $3(3.4 \%)$ & 0 & 0 \\
Skin loss & $1(1.1 \%)$ & 0 & 0 \\
Hematoma & 0 & 0 & 0 \\
\hline
\end{tabular}

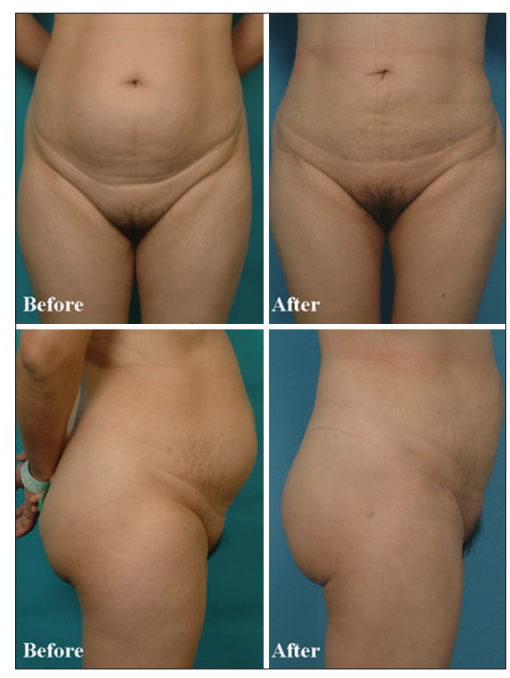

Figure 3: Frontal and lateral images of forty-year-old female patient with abdominal protrusion before and 66 months follow-up, post-endoscopic abdominoplasty. 


\section{DISCUSSION}

Most Asian women who undergo endoscopic abdominoplasty procedures fall under the post-pregnancy category, finding their abdominal area to have been overstretched during the prenatal period. Basic cases of patients with this post-pregnancy stretched muscle/skin tissue fall under four types as mentioned in Methods. Type A - for which our study's patients categorized under - is where stretching occurs in the junction of the rectus sheath at the center of the abdominal area and central plication of the rectal sheath can be performed.

Through operative procedures, besides suprapubic incision, endoscopic incisions can be made for past surgical procedures like appendectomy, cesarean section, and laparotomy. Such cases administer the endoscope to reenter through the previously incised area of the existing scar. At the umbilical section, incisions can also be made in different forms: Y-shape, Z-shape, periumbilical and vertical. Through the umbilical incision, the skin flap, in conjunction with subcutaneous fatty tissue, is undermined and dissected for prevention of ischemic change of umbilical flap after reconstruction.

The most difficult part of the endoscopic technique is the dissection, requiring the maximum effort and attention of the assistant, and even a second assistant for obtaining good exposure by lifting the abdominal wall. It was observed that the electrcautery was more applicable and faster than using the traditional dissection technique, and coagulation of the bleeder could be safely conducted. However arterial or active bleeding should be ligated for prevention of complications. During abdominal wall dissection, once perforator vessels were cauterized or cut, instances of tissue ischemia or even skin necrosis can result. In our series, none of our subjects showed such responses. ${ }^{[15-17]}$

The second most difficult part of endoscopic abdominoplasty is the repair of the fascia. When such an application was performed, elliptical marking on the external rectal sheath about $12-15 \mathrm{~cm}$ apart was made to indicate the extent of the tissue in which those instruments were intended to hold together.

It is important to note that following the above procedure, our study included 16 patients $(18.1 \%)$ that received power aspirator liposuction treatment as a form of adjunctive therapy to address body contouring. This procedure was usually performed before endoscopic abdominoplasty with the administration of a topical tumescent. An extra procedure of transaxillary-approached-augmentation mammoplasty for two patients (2.3\%) was done after endoscopic abdominoplasty and adjusted body contour easily.

Arterial or active bleeding is one of the factors that must be observed and considered intraoperatively. Through proper patient preparation, when ligation or coagulation of the bleeder can be safely conducted, fibrin sealant can be actively administered. Certain precautions that are to be taken when applying fibrin sealant for treatment are limited, but nonetheless, contain important procedural steps in assuring the prime safety of the patient. Awareness of the tissue samples that are exchanged and/or transferred amongst and between patients is crucial in preventing the occurrence of infection. Such invasive procedures do stand at risk for experiences of hypersensitivity and allergic and/or anaphylactic reactions as well. ${ }^{[18-23]}$ However, these complications were not observed in our series.

Advantages of endoscopic surgery include, but are not limited to the decreased incidence of scarring, bleeding, numbness, and/or edema. One major advantage of using the endoscope for abdominoplasty that our study observed is minimization of scarring. Such administration of this method minimized scar observation, and also pain sensations that commonly result from elongated incisions, was not apparent in our study. From the minimally invasive endoscope incision, discomfort and even numbness was sufficiently diminished. ${ }^{[16]}$ Disadvantages arose when large vessels were approached with the endoscope, in which procedures for vessel ligation, as opposed to cutting, were administered. However, use of the endoscope posed a deterrent in the limited visual field and operation region. For future studies, it is important to note that when handling large vessels, steps to address the issue of intensified bleeding can be avoided when proper use of endoscope is in place.

Technical considerations for videoendoscopic assisted abdominoplasty begin with the creation of a sizeable surgical space on patients for accessible procedures. Other important points to keep in mind are hemostasis, smoke filtering, fluid removal, scope care and upkeep, and steady positioning of electrode tip. It is also especially important to achieve a high level of hand-eye coordination and maximum efficiency and attention during surgeries. Other quality standards that must also receive attention include selecting an experienced surgeon in standard "open abdominoplasty" for the quality of muscle and fascia repair must be equivalent to that obtained during the "open abdominoplasty" technique. No other scars are to be present on the abdomen. Care of these tools and techniques are not to be taken lightly. Users must focus on the aforementioned points to achieve the maximum outcome and results for both surgeon and patient.

\section{CONCLUSION}

Advantages of standard endoscopic surgery include diminished incidence of scarring, less numbness, bleeding and edema. The major advantage of endoscopic surgery in the abdominal area is the minimization of scarring. This advantage is preferable to the traditional suprapubic approach, 
as the incidence of long term scarring is lessened. Thus, like all innovative procedures, there is a learning curve to be followed in order to achieve the optimal technical expertise necessary to yield the best results. For this to happen, new training and instrumentation is required, and the limitations of this technique must be acknowledged. Combining fibrin sealant and endoscope surgery can achieve optimal results, however, more patient data is needed for a better outcome and a more standardized result. Once future cases are studied, the combination of the endoscopic technique with fibrin sealant will be able to produce significant results in an optimum surgical time frame.

\section{REFERENCES}

1. Carson WG Jr. Arthrosocopy of the shoulder, anatomy and technique. Orthop Rev 1992;21:143-53.

2. Grimes D. Frontiers of the operative laparoscopy: A review and critique of the evidence. Am J Obstet Gynecol 1992;166:1062-71.

3. Gadacz TR. U.S. Experience with laparoscopic cholecystectomy. Am J Surg 1993;165:450-4.

4. Sasaki GH. Tissue expansion. In: Jurkiewicz MJ, Krizek TJ, Maths SJ, Aryian S, editors. Plastic surgery-principles and practice. St. Louis: Mosby; 1990. p. 1609-34.

5. Chow JC. Endoscopic release of the carpal ligament: A new technique for carpal tunnel syndrome. Arthroscopy 1989;5:19-24.

6. Liang M, Narayanan K. Endoscopic ablation of the frontalis and corrugator muscles: A clinical Study. Plast Surg Forum 1992;15:54.

7. Ramirez OM. The subperiosteal rhytidectomy: The third generation face lift. Ann Plast Surg 1992;28:218-32.

8. McKinney P, Mossie RD, Zwkowski ML. Criteria for the forehead lift. Aesth Plast Surg 1991;15:141-7.

9. Chang CJ. Combining the $\mathrm{CO}_{2}$ laser and the endoscope to remove soft tissue masses from the forehead area. Photomed Laser Surg 2005;23:509-12.
10. Ho LY. Endoscopic assisted transaxillary augmentation mammoplasty. Br J Plast Surg 1993;46:332-6.

11. Faria Correa MA. Videoendoscopic subcutaneous techniques for aesthetic and reconstructive platic surgery. Plast Reconstr Surg 1995;96:446-53.

12. Matarasso A. Minimal-access variations in abdominoplasty. Ann Plast Surg 1995;34:255-63.

13. Zukowski ML, Ash K, Spencer D, Malanoski M, Moore G. Endoscopic intracorporal abdominoplasty; a review of 85 cases. Plast Reconstr Surg 1998;102:516-27.

14. Aly A, Avila E, Cram AE. Endoscopic plastic surgery. Surg Clin North Am 2000;80:1373-82.

15. Ousterhout KD. Combined suction assisted lipectomy, surgical lipectomy and surgical abdominoplasty. Ann Plast Surg 1990;24:126-32.

16. Christman KD. Death following suction lipectomy and abdominoplasty. Plast Reconstr Surg 1986;78:428.

17. Stewart KJ, Stewart DA, Coghlan B, Harrison DH, Jones BM, Waterhouse N. Complications of 278 consecutive abdominoplasties. J Plast Reconstr Aesthet Surg 2006;59:1152-5.

18. Grey EG. Fibrin as a hemostatic in cranial surgery. Surg Gynecol Obstet 1915;21:452.

19. Dresdale A, bowman Jr FO, Malm JR. Hemostatic effectiveness of fibrin glue derived from single-donor fresh frozen plasma. Ann Thorac Surg 1985;40:385-7.

20. Bronestedt S, Rank F, Olson PS. Wound healing and formation of granulation tissue in normal and defibrinogenerated rabbits. Eur Surg Res 1980;12:12-21.

21. Marchac D, Sandor G. Face lifts and sprayed fibrin glue: An outcome analysis of 200 patients. Br J Plast Surg 1994;47:306-9.

22. Mandel MA. Closure of blephaloplasty incisions with autulogous fibrin glue. Arch Ophthalmol 1990;108:842-4.

23. Marchac D, Ascherman J, Arnaud A. Fibrin glue fixation in forehead endoscopy: Evaluation of our experience with 206 cases. Plast Reconstr Surg 1997;100:704-12. 